\title{
España y Venezuela como impulsores de la academia contable colombiana
}

\author{
Hugo Arlés Macías ${ }^{a}$
}

Información del artículo
Recibido:03/07/2019
Aceptado: 12/09/2019
Clasificación JEL
M40
Este es un artículo Open Access bajo
la licencia CC BY NC [https://creative-
commons.org/licenses/by-nc/4.0]
Enlace Dol
https://dx.doi.org/10.24142/rvc.n20a1
Sugerencia de citación
- Macías,H.A.(2019).España y
Venezuela como impulsores de la
academia contable colombiana.
Revista Visión Contable, 20, 8-20.
doi: 10.24142/rvc.n20al

Información del artículo

Recibido:03/07/2019

Aceptado: 12/09/2019

Clasificación JEL

la licencia CC BY NC [https://creativecommons.org/licenses/by-nc/4.0]

Enlace DO

do: $10.24142 / 7 v a n 201$

\section{Spain and Venezuela as drivers of the Colombian accounting} academy

\section{Resumen}

El objetivo de este trabajo es destacar el papel que han jugado, recientemente, dos programas de doctorado y un pequeño grupo de académicos de España y Venezuela, en la cualificación de la academia contable colombiana. Profesores de diferentes ciudades se han vinculado como estudiantes a estos doctorados y han empezado a tejer redes que les permiten participar en discusiones más exigentes, tanto a nivel de congresos, como de publicaciones. La masa crítica que se está formando permitirá que, en la tercera década del siglo, la academia colombiana participe activamente en las discusiones extrarregionales de la frontera del conocimiento en contabilidad.

Abstract
The objective of this work is to highlight
the role that two doctoral programs and
a small group of academics from Spain
and Venezuela have recently played in
the qualification of the Colombian ac-
counting academy. Teachers from diffe-
rent cities have been linked as students
to these doctorates and have begun to
weave networks that allow them to par-
ticipate in more demanding discussions,
both at the congress level and in publi-
cations. The critical mass that is being
formed will allow, in the third decade of
the century, the Colombian academy to
actively participate in the extra-regional
discussions of the knowledge frontier in
accounting.
Key words
Accounting Academy, publications,
frontier of knowledge

Academia contable, publicaciones, frontera del conocimiento

a Profesor asociado de la Universidad de Medellín; coordinador de la Maestría en Tributación y Política Fiscal. Correo electrónico: hmacias@udem.edu.co 
La academia contable colombiana terminó la segunda década del siglo con algunos avances visibles y con varios retos pendientes. Las discusiones locales, centradas en lo epistemológico, han avanzado hacia trabajos empíricos y a unas relaciones emergentes más allá de Hispanoamérica. En la segunda década del siglo, la formación de algunos académicos contables colombianos les permitió mejorar el rigor de sus investigaciones, con lo cual empezaron a participar como ponentes en eventos más exigentes y dieron los primeros pasos en cuanto a publicación en revistas extrarregionales.

En este documento corto se quieren presentar dos experiencias que no han sido destacadas antes en las revistas contables colombianas: el impulso que se ha dado a la creación de capacidades académicas en Colombia, desde Venezuela y España. Ese impulso se ha dado desde unas instituciones en particular, con el liderazgo de académicos claramente identificables. Concretamente, se hará referencia a los aportes de dos programas de doctorado con base en esos países, y a un grupo de académicos que han hecho posible que se amplíen las capacidades nacionales.

\section{Venezuela como impulsor de la investigación contable colombiana}

En la segunda década de este siglo, un grupo importante de académicos de diferentes ciudades colombianas inició sus estudios en el Doctorado en Ciencias Contables de la Universidad de los Andes, en Mérida, Venezuela. Ese programa académico, liderado por la profesora Norka Viloria, permitió a los estudiantes adentrarse en las profundidades de la contabilidad, al incursionar en obras como las de Anthony Hopwood (Agudelo-Vargas, 2013), Norman Macintosh (Franco-Ruiz, 2013), Jürgen Habermas (Gracia-López, 2013), Richard Laughlin (Jiménez-Aguirre, 2013) o Wai Fong Chua (Vargas-Restrepo, 2013). Huellas importantes del nivel de profundidad alcanzado quedaron impresas en el número 14 de la Revista Lúmina, publicado en el año 2013.

Pero, además de incursionar en las obras de académicos contables con alto reconocimiento internacional, que el profesor Keith Hooper (2014) denomina "grandes nombres", también el programa de doctorado ha permitido a sus participantes aumentar el rigor y nivel de profundidad en líneas de investigación que se venían trabajando. Por ejemplo, el profesor Marco Machado (2016) ha fortalecido su línea de trabajo sobre modelos conta- 
bles y el profesor Héctor Darío Betancur $(2014 ; 2017)$ avanzó en su línea de trabajo sobre sistemas contables. Asimismo, la profesora Sandra Perea-Murillo $(2014 ; 2015 a)$ desarrolló su tesis doctoral sobre deterioro del valor de los activos, dando continuidad a trabajos en temas similares, como el valor razonable (Perea-Murillo, 2015b). Por su parte, la profesora Faustina Manrique (2018) escribió una tesis sobre educación contable, y el profesor Esteban Martínez-Salinas (2019) se basó en la obra de Foucault para proponer nuevas aplicaciones a la contabilidad gubernamental. En contraste, el profesor Edilgardo Loaiza-Betancur (2014a; 2014b), primer egresado colombiano del doctorado, prefirió desarrollar una nueva línea, al escribir su tesis doctoral sobre el ejercicio de la profesión y sus efectos sobre la salud del profesional contable. Si se estudia la trayectoria de cada uno de estos académicos puede detectarse, claramente, el valor agregado del Doctorado en Ciencias Contables de la Universidad de los Andes.

\begin{tabular}{|c|c|c|c|}
\hline Nombre & Filiación institucional 2019 & Ciudad & Año de egreso \\
\hline Edilgardo de Jesús Loaiza Betancur & $\begin{array}{l}\text { Politécnico Colombiano Jaime } \\
\text { Isaza Cadavid }\end{array}$ & Medellín & 2014 \\
\hline Carlos Mario Vargas Restrepo & Universidad Católica de Oriente & Rionegro & 2016 \\
\hline Marco Antonio Machado Rivera & Universidad de Antioquia & Medellín & 2016 \\
\hline María Victoria Agudelo Vargas & Universidad Católica Luis Amigó & Medellín & 2017 \\
\hline Rubiela Jiménez Aguirre & Universidad de Manizales & Manizales & 2017 \\
\hline Sandra Patricia Perea Murillo & $\begin{array}{l}\text { Universidad Tecnológica del } \\
\text { Chocó }\end{array}$ & Quibdó & 2017 \\
\hline Sergio Agustín Suárez Nieves & Distrito de Cartagena & Cartagena & 2017 \\
\hline Héctor Darío Betancur & Universidad de Medellín & Medellín & 2018 \\
\hline Esteban Segundo Martínez Salinas & $\begin{array}{l}\text { Universidad Autónoma de } \\
\text { Colombia }\end{array}$ & Bogotá & 2019 \\
\hline Faustina Manrique Ramírez & Universidad Libre de Colombia & Bogotá & 2019 \\
\hline \multicolumn{4}{|c|}{ Fuente: elaboración propia con base en CvLac y certificado oficial de la Universidad de los Andes. } \\
\hline
\end{tabular}

Buena parte de los académicos que se incorporaron a ese programa doctoral en Venezuela hacen parte de la tradición contable colombiana, que lleva varias décadas de desarrollo. Allí fortalecieron una línea de trabajo que ya venían construyendo y también vincularon de manera rigurosa a otros académicos contables más jóvenes. La participación en ese programa 
fue el primer proceso colectivo de cualificación en el que ha participado un número amplio de académicos colombianos, de diferentes ciudades.

Por otro lado, en el año 2010, migró a Colombia una contadora pública (y economista) venezolana, que rápidamente se convirtió en un eslabón importante de cualificación para la academia contable de nuestro país. Se trata de la Dra. Mary Vera-Colina, quien después de trabajar 16 años en la Universidad del Zulia, en Maracaibo (Venezuela), se vinculó a la Universidad Nacional de Colombia, sede Bogotá. Desde allí, ha venido liderando la construcción de una red internacional de académicos contables que está en proceso de consolidación. Entre sus múltiples actividades, la profesora Vera-Colina ha liderado congresos en Colombia y ha participado en la organización de otros encuentros en países como Brasil e Inglaterra, donde se han vinculado académicos contables colombianos con sus pares no hispanoparlantes.

Después de abordar distintos temas, en 2016, el “Tercer Congreso Global en Contabilidad y Finanzas” (INTERGES) se centró en la perspectiva crítica en contabilidad (también en finanzas y gestión). Esta versión del evento bienal contó con la presencia de académicos muy influyentes en la contabilidad crítica. Una de ellas es la profesora británica Christine Cooper, coeditora actual de la revista Critical Perspectives on Accounting (CPA) y miembro de los consejos editoriales de revistas como Accounting, Organisations and Society (AOS), Accounting, Auditing and Accountability Journal (AAAJ), Accounting Forum (AF) y Sustainability Accounting Management and Policy Journal (SAMPJ). En ese mismo evento, estuvo el profesor David Cooper, quien fue fundador, en 1990, de la revista Critical Perspectives on Accounting y coeditor de la misma (con Tony Tinker) entre 1990 y 2008; él también hace parte del equipo editorial de AOS y AF, entre otras. Igualmente, participó el profesor australiano Medhat Endrawes, coautor de Australian Accounting Review (Arthur, Endrawes y Ho, 2017) y de Accounting \& Finance (Endrawes, Feng, Lu y Shan, 2018); él ha hecho visitas posteriores a grupos de trabajo contables en universidades de Barranquilla, Bogotá, Bucaramanga, Medellín, Tunja y otras ciudades latinoamericanas.

Ese encuentro de 2016, en Bogotá, tuvo continuidad, entre otros, en el "Qualitative Research and Critical Accounting: A South American Workshop", que se llevó a cabo en la Universidad de Sao Paulo (Brasil), a finales de 2018. Allí, la profesora Vera-Colina participó activamente en la organización, de la mano de las profesoras Silvia Casa Nova (USP), Fernanda Filgueiras Sauerbronn (UFRJ) y Elisabeth de Oliveira Vendramin (UFMS). Este evento contó con la presencia de Yves Gendron, como invi- 
tado especial, quien, además de ser una autoridad internacional en contabilidad crítica, es uno de los tres editores actuales de Critical Perspectives on Accounting. También, la profesora Vera-Colina presentó varias ponencias y consiguió que la Universidad Nacional de Colombia fuera la sede de la versión siguiente del evento, "Qualitative Research and Critical Accounting 2019: A Latin American Conference", convocado por la revista CPA.

Antes de que se llevara a cabo el evento de 2019, en Bogotá, se desarrolló una conferencia en Inglaterra donde la profesora Vera-Colina, en compañía de las profesoras Casa Nova y Filgueiras (ambas de Brasil), lideró una mesa con participación latinoamericana. Se trató de una mesa de estudios críticos en contabilidad, en el marco de la conferencia internacional anual número 11 de Critical Management Studies (CMS). El evento se desarrolló la última semana de junio de 2019, en la ciudad británica de Milton Keynes, con participación de ponencias provenientes de varias regiones de Colombia y de Brasil, así como un par de trabajos de Argentina y Chile. Allí, participó de nuevo Yves Gendron y se fortalecieron los lazos de la red latinoamericana con la profesora Jill Atkins de Sheffield University, la cual, en compañía de la escuela de negocios de la Universidad de Manchester, fue reconocida por el profesor Richard Baker (2011, p. 214) como la cuna de la investigación contable crítica, el espacio donde se creó esta corriente de investigación. La profesora Vera-Colina ha liderado una red abierta, internacional y plural, que está creciendo y tiene un futuro muy prometedor. Esa capacidad de armar equipos de trabajo para tejer redes la adquirió en su primera etapa profesional en Venezuela.

\section{España como impulsor de la investigación contable colombiana}

Por la misma época que un grupo de académicos contables colombianos inició sus estudios doctorales en la Universidad de los Andes (Venezuela), el profesor Mauricio Gómez Villegas comenzó sus estudios en el Doctorado en Contabilidad y Finanzas de la Universidad de Valencia, en España. Al principio, este doctorado era un caso aislado, como el de otros académicos contables que adelantaron estudios de posgrado en el exterior. Pero pocos años después empezaron a vincularse al mismo programa un pequeño grupo de profesores de Bogotá, Cali y Medellín, que consiguieron terminar sus estudios doctorales antes de finalizar la segunda década del 
siglo, como puede apreciarse en la Tabla 2. El profesor Gómez Villegas es el académico contable colombiano con mayor impacto de sus publicaciones (Macías, 2016) y abrió caminos en términos de formación de alto nivel en contabilidad.

Tabla 2. Primeros siete colombianos egresados del Doctorado en Contabilidad y Finanzas Corporativas, de la Universidad de Valencia, España

\begin{tabular}{|l|l|l|c|}
\hline \multicolumn{1}{|c|}{ Nombre } & \multicolumn{1}{|c|}{ Filiación institucional 2019 } & Ciudad & $\begin{array}{c}\text { Año de } \\
\text { egreso }\end{array}$ \\
\hline Mauricio Gómez Villegas & Universidad Nacional de Colombia & Bogotá & 2013 \\
\hline José ObdulioCurveloHassán & Universidad Cooperativa de Colombia & Bogotá & 2017 \\
\hline Julieth Emilse Ospina Delgado & Pontificia Universidad Javeriana & Cali & 2017 \\
\hline Yaismir Adriana Rivera Arrubla & Universidad del Valle & Cali & 2017 \\
\hline Jaime AndrésCorreaGarcía & Universidad de Antioquia & Medellín & 2018 \\
\hline Yuli MarcelaSuárezRico & Universidad Central & Bogotá & 2018 \\
\hline Jeimi MaribelLeónSilva & Reincorporación a partir de 2020 & Bogotá & 2019 \\
\hline
\end{tabular}

Fuente: elaboración propia con base en CvLac e información suministrada por Vicente Ripoll y Jeimi León.

Esta iniciativa, que al principio fue aislada y a la que luego se le fueron sumando otros académicos de diferentes ciudades, se convirtió en un proceso colectivo con participación de un número amplio de estudiantes provenientes de un número mayor de ciudades. La explicación central del crecimiento en la cantidad de estudiantes colombianos de ese programa está en la promoción deliberada del mismo en distintas regiones del país por parte del profesor Vicente Ripoll Feliu, de la Universidad de Valencia, quien encontró apoyo en directivos de programas contables de diferentes regiones de Colombia, con los que se lograron los apoyos institucionales que permitieran incrementar el número de estudiantes del doctorado (Tabla 3 ).

A la gestión directa que el profesor Vicente Ripoll ha hecho en ciudades como Armenia, Barranquilla, Cartagena y Medellín, se suma el prestigioso grupo de profesores del doctorado que ofrecen los distintos seminarios. Entre ellos, además de Ripoll, están Begonia Giner, Belén Gil de Albornoz, Chelo Pucheta, Cristina de Fuentes, David Naranjo, Ernesto López Valeiras, José García, José López y Miguel Arce Gisbert. Estos profesores se destacan por sus ponencias y, especialmente, por sus artículos en las discusiones internacionales de contabilidad, particularmente entre la comunidad académica que se comunica en inglés. La Dra. Giner, además de ser profesora 
y directora del doctorado, fue presidente de la European Accounting Association (EAA), en el período 2011-2013, es reconocida internacionalmente por sus trabajos sobre reportes corporativos y, desde 2019, es la directora del Comité de Reporte Corporativo de la EAA.

También es importante destacar el papel que han jugado profesores como Vicente Montesinos Julve y Maria Antonia García-Benau, quienes han dirigido varias tesis de los doctores colombianos. Además del reconocimiento que ellos han ganado por su trayectoria, introdujeron a sus estudiantes en las discusiones internacionales formales a través de participación en eventos y de publicaciones en importantes revistas internacionales. Las primeras publicaciones con los directores de tesis son del profesor Mauricio Gómez, sobre la contabilidad gubernamental en Colombia (Gómez-Villegas y Montesinos, 2012) y sobre el rol de las IPSAS en el sector público latinoamericano (Brusca, Gómez-Villegas y Montesinos, 2016). Varias de las publicaciones del profesor Gómez analizan realidades regionales; su objeto de estudio ya no es solo Colombia, sino la región.

Por su parte, la profesora María Antonia García-Benau ha dirigido tesis de estudiantes de Bogotá, Cali y Medellín, en compañía de otros codirectores. Sus primeras estudiantes dirigidas fueron las profesoras Julieth Ospina y Adriana Rivera, de la Universidad del Valle (Cali), ambas orientadas con la profesora Ana Zorio-Grima, de la Universidad de Valencia. Con la profesora Ospina construyeron una tesis sobre cursos masivos y en línea (MOOC), tanto desde la oferta universitaria (Ospina-Delgado, Zorio-Grima y García-Benau, 2016) como de su contribución a la formación en IFRS (Ospina-Delgado, García-Benau y Zorio-Grima, 2016), y como una innovación en general (Ospina-Delgado y Zorio-Grima, 2016). Con la profesora Rivera escribieron una tesis sobre reportes integrados, que incluyó aspectos conceptuales (Rivera-Arrubla, Zorio-Grima y García-Benau, 2016), la conectividad con redes sociales (Rivera-Arrubla y Zorio-Grima, 2016) y factores explicativos (Rivera-Arrubla y García-Benau, 2017)(Ver tabla 3).

La profesora García-Benau también se asoció con Emma Garcia-Meca, para dirigir la tesis de Jaime Correa sobre grupos empresariales y reportes de sostenibilidad en América Latina. Desde allí, publicaron un primer artículo sobre estrategias de comunicación de los reportes corporativos (Correa-Garcia, Garcia-Benau y Garcia-Meca, 2018) y construyeron un plan para seguir trabajando más allá de la tesis. En un sentido similar, la profesora García-Benau se asoció con el profesor Mauricio Gómez Ville- 


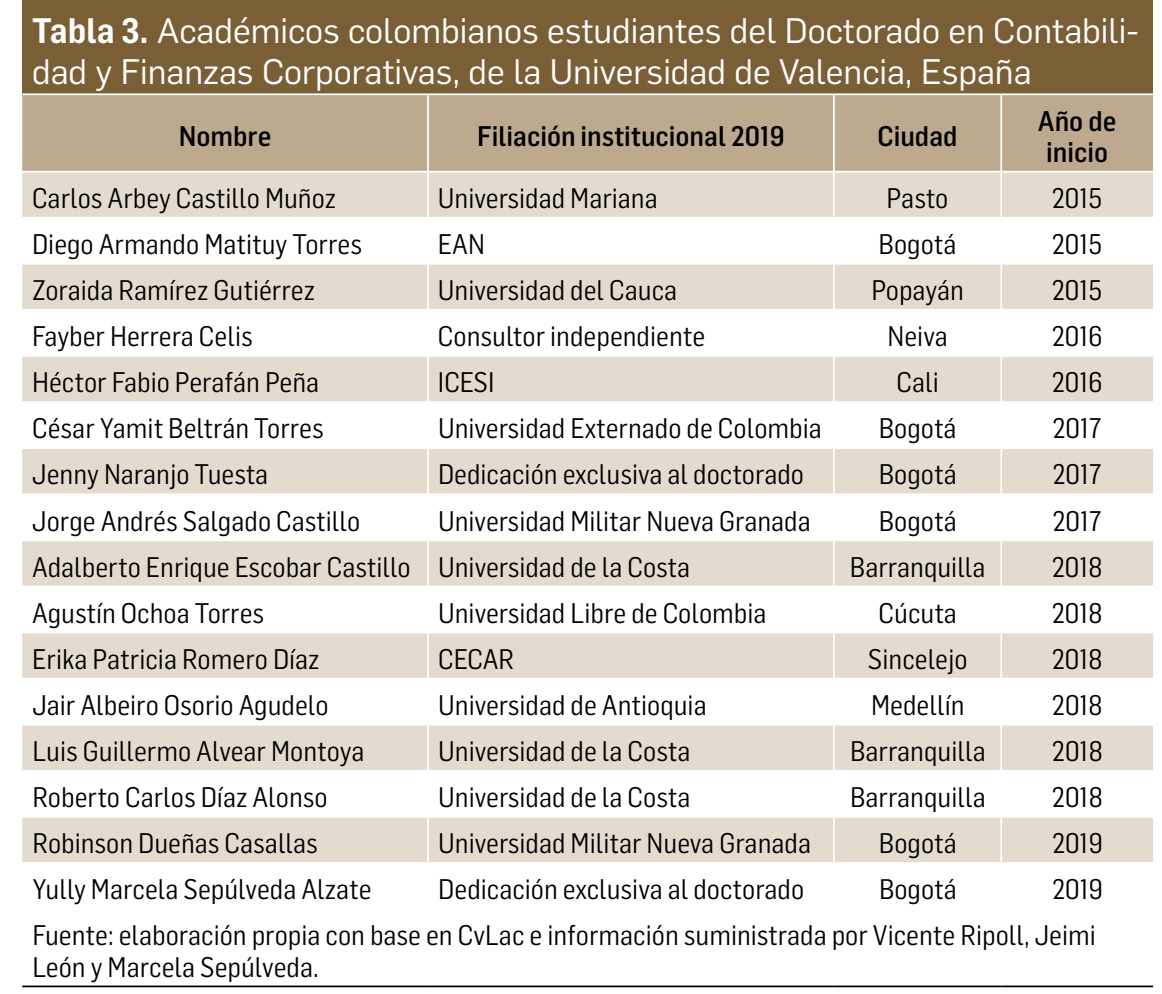

gas para dirigir la tesis de Marcela Suárez, sobre comunicación de la responsabilidad social corporativa en redes sociales. Consiguieron publicar dos trabajos iniciales sobre las comunicaciones vía Twitter (Suárez-Rico, Gómez-Villegas y García-Benau, 2018) y sobre las comunicaciones vía Facebook (Suárez-Rico, García-Benau y Gómez-Villegas, 2019), en empresas latinoamericanas.

El trabajo de María Antonia García-Benau es particularmente valioso porque ha tejido relaciones académicas con profesores colombianos de varias ciudades, siempre se ha hecho acompañar de profesores que hacen parte de su red de trabajo, está asesorando otras tesis en construcción y ya empezó a viajar a Colombia a encontrarse con diferentes miembros de la comunidad nacional que frecuentan menos los espacios europeos, entre otras razones. Una muy importante es que ella ha contribuido bastante a romper el mito de que es imposible publicar en prestigiosas revistas internacionales, y lo ha conseguido con autores colombianos de varias ciudades y, además, en varios 
casos los artículos están disponibles en internet con acceso abierto.

También es necesario destacar el papel que ha desempeñado Vicente Ripoll Felieu. Sus sucesivos viajes a Colombia (y a otros países de América Latina) le han permitido construir relaciones académicas personales con un grupo amplio de integrantes de la comunidad contable nacional. Pero más allá de la amistad y la participación en congresos en diferentes países de la región, ha construido caminos de cualificación, facilitando institucionalmente que un número amplio de académicos colombianos acceda a formación de alto nivel, guiados por un grupo de los académicos contables internacionales más influyentes. El profesor Ripoll es, sin duda, protagonista de este proceso y merece todo el reconocimiento por su trabajo.

Igualmente, es visible el papel de profesores como Vicente Montesinos, quien ha dirigido varias tesis doctorales, Ana Zorio-Grima y Emma García-Meca, quienes están conformando equipos de trabajo con académicos colombianos que conducirán a importantes contribuciones futuras, con participación directa desde este país. Por supuesto, los estudiantes de los doctorados están haciendo sus propios esfuerzos académicos, familiares, personales y económicos, que redundarán en la conformación de una masa crítica que pueda participar a futuro ampliamente en las discusiones de frontera en contabilidad.

La dinámica que se empezó a tejer en el doctorado de la Universidad de los Andes, en Venezuela, no es de menor envergadura. Allí también es necesario reconocer y valorar el trabajo de la profesora Norka Viloria, quien impulsó un proceso con pocos antecedentes en la región, en medio del escepticismo, y quien consiguió, desde el principio, una participación amplia de académicos colombianos de diferentes regiones del país, en un programa con alto nivel de rigor. Aunque no es fácil de lograr, lo consiguió por su cercanía con la academia contable colombiana, sus visitas frecuentes y su gran capacidad para tejer redes académicas, formalizarlas y mantenerlas en el tiempo. Asimismo, en el futuro cercano, la academia contable colombiana logrará establecer vínculos duraderos y más visibles, entre su tradición de varias décadas y la dinámica internacional de la contabilidad crítica, gracias a las redes que la profesora venezolana Mary Vera Colina está liderando, especialmente con Brasil, Canadá y Reino Unido.

Si bien los dos programas de doctorado mencionados se convirtieron en los vehículos más importantes de cualificación colectiva de la academia contable colombiana en la segunda década del siglo, no son los únicos espacios. Un grupo (pequeño) de profesores ha estudiado doctorados conta- 
bles de alta calidad en países como Argentina, Brasil, Inglaterra y en otras universidades españolas. También se ha contado con el acompañamiento de profesores de diferentes universidades extranjeras que han contribuido visiblemente a la cualificación de académicos contables en Colombia. En todo caso, la tercera década del siglo será la de la vinculación de los académicos contables colombianos a las discusiones de frontera en contabilidad. 


\section{Referencias}

Agudelo-Vargas, M. V. (2013). Análisis de los aportes de Anthony G. Hopwood a la disciplina contable. Revista Lúmina, 14, 290-315.

Arthur, N., Endrawes, M. y Ho, S. (2017). Impact of partner change on audit quality: An analysis of partner and firm specialisation effects. Australian Accounting Review, 27(4), 368-381.

Baker, C. R. (2011). A genealogical history of positivist and critical accounting research. Accounting History, 16(2), 207-221.

Betancur, H. D. (2014). El sistema contable en perspectiva sistema-entorno. Revista Lúmina, 15, 202 225.

Betancur, H. D. (2017). Elementos reflexivos para un análisis sistémico-constructivista de la noción "sistema contable". Visión Contable, 15, 10-33.

Brusca, I., Gómez-Villegas, M. y Montesinos, V. (2016). Public financial management reforms: The role of IPSAS in Latin-America. Public Administration and Development, 36(1), 51-64.

Correa-Garcia, J. A., Garcia-Benau, M. A. y Garcia-Meca, E. (2018). CSR communication strategies of colombian business groups: An analysis of corporate reports. Sustainability, 10(5), 1-19.
Endrawes, M., Feng, Z., Lu, M. y Shan, Y. (2018). Audit committee characteristics and financial statement comparability. Accounting \& Finance, 1-35. doi:https://doi. org/10.1111/acfi.12354

Franco-Ruiz, R. (2013). Aportes del posestructuralismo y el posmodernismo al desarrollo del pensamiento contable. La perspectiva de Norman Macintosh. Revista Lúmina, 14, 54-87.

Gómez-Villegas, M. y Montesinos, V. (2012). Las innovaciones en contabilidad gubernamental en latinoamérica: el caso de Colombia. Innovar, 22(45), 17-35.

Gracia-López, E. (2013). Habermas y su incidencia en la contabilidad. Revista Lúmina, 14, 88-115.

Hooper, K. (2014). "What's in a name": or why create. Qualitative Research in Accounting \& Management, 11(1), 8-12.

Jiménez-Aguirre, R. (2013). Un aporte de Richard Laughlin a la contabilidad: El pensamiento de Middle-Range. Revista Lúmina, 14, 176-199.

Loaiza-Betancur, E. (2014a). Modelo del costo de alcanzar el éxito. Una valoración desde la exitodinámica y la incidencia en la salud del Contador Público colombiano (Tesis de doctorado). Mérida, Venezuela. 
(2014b). El ejercicio de la profesión contable en Colombia. Una mirada desde el paradigma contable del comportamiento basado en la conducta y el deterioro de la salud física y mental. En-Contexto, 2, 147-164.

Machado, M. A. (5 de octubre de 2016). Las creencias compartidas en el discurso sobre modelo contable. Trabajo presentado en XXI Congreso Internacional de Contaduría, Administración e Informática. Recuperado de http://congreso. investiga.fca.unam.mx/docs/xxi/ docs/5.05.pdf

Macías, H. A. (2016). Visibilidad de la investigación contable colombiana en Google Académico. Contexto, 5, 3-6.

Manrique, F. (2018). Los cuerpos dóciles: el caso de la educación contable. Revista Científica General José María Córdova, 16(24), 131-153.

Martínez-Salinas, E. S. (2019). Evaluación de la teoría del poder de Foucault en el control sobre la información contable gubernamental (Tesis de doctorado). Mérida, Venezuela.

Ospina-Delgado, J. E., García-Benau, M. A. y Zorio-Grima, A. (2016). Massive Open Online Courses for IFRS education: a point of view of Spanish Accounting Educators. Procedia-Social and Behavioral Sciences, 228, 356-361.
Ospina-Delgado, J. y Zorio-Grima, A. (2016). Innovation at universities: A fuzzy-set approach for MOOC-intensiveness. Journal of Business Research, 69(4), 1325-1328.

Ospina-Delgado, J. E., Zorio-Grima, A. y García-Benau, M. A. (2016). Massive open online courses in higher education: A data analysis of the MOOC supply. Intangible Capital, 12(5), 1401-1450.

Perea-Murillo, S. P. (2014). Deterioro del valor de activos a la luz de la Teoría del Valor. Revista Lúmina, 15, 130-145.

(2015a). Reflexión epistemológica en torno al concepto deterioro del valor: análisis de sus efectos en la información financiera. Teuken Bidikay, 6, 63-79. (2015b). Perspectiva crítica del valor razonable en el marco de la crisis financiera. Cuadernos de Contabilidad, 16(42), 761-779.

Rivera-Arrubla, Y. A. y García-Benau, M. A. (2017). Integrated reports: disclosure level and explanatory factors. Social Responsibility Journal, 13(1), 155-176.

Rivera-Arrubla, Y. A. y Zorio-Grima, A. (2016). Integrated reporting, connectivity, and social media. Psychology \& Marketing, 33(12), 1159-1165.

Rivera-Arrubla, Y. A., Zorio-Grima, A. y García-Benau, M. A. (2016). El concepto de informe integrado 
como innovación en reporting corporativo. Journal of Innovation \& Knowledge, 1(3), 144-155.

Suárez-Rico, Y. M., García-Benau, M. A. y Gómez-Villegas, M. (2019). CSR communication through $\mathrm{Fa}$ cebook in the Latin American integrated market (MILA): Disclosure, interactivity and legitimacy. Meditari Accountancy Research, 27(4). doi:10.1108/MEDAR-03-2018-0313

Suárez-Rico, Y. M., Gómez-Villegas, M. y García-Benau, M. A. (2018). Exploring Twitter for CSR Disclosure: Influence of CEO and firm characteristics in Latin American companies. Sustainability, 10(8), 1-19.

Vargas-Restrepo, C. M. (2013). Caracterizacion de la investigacion contable. El aporte de Chua. Revista Lúmina, 14, 262-289. 